\title{
Coracobrachialis muscle pierced by two nerves: case report
}

\author{
Dawit Habte Woldeyes, Belta Asnakew Abegaz \\ Department of Human Anatomy, College of Medicine and Health Sciences, Babir Dar University, Babir Dar, Ethiopia
}

\begin{abstract}
The brachial plexus is formed by the union of the ventral rami of C5-T1. Contributions to the plexus by $C 4$ and $\mathrm{T} 2$ differ. The coracobrachialis muscle is an elongated muscle in the superomedial part of the arm. It is a useful landmark for locating other structures in the arm. Variations exist to the coracobrachialis muscle, and to the formation of the brachial plexus, its terminal branches and its relation to surrounding structures. In this report, the coracobrachialis muscle is pierced by two nerves, and one of these nerves joins the median nerve after piercing the coracobrachialis muscle. Awareness of the possible variations of brachial plexus and its surrounding structures is necessary for adequate clinical, surgical and radiological management.
\end{abstract}

Keywords: brachial plexus; coracobrachialis; median nerve; musculocutaneous nerve; unnamed nerve

Anatomy 2016;10(2):148-152 @2016 Turkish Society of Anatomy and Clinical Anatomy (TSACA)

\section{Introduction}

The brachial plexus is formed by the union of the ventral rami of $\mathrm{C} 5-\mathrm{T} 1$. The most common arrangement of the brachial plexus is as follows: the fifth and sixth rami unite as the upper trunk, the eighth cervical and first thoracic rami join as the lower trunk, the seventh cervical ramus becomes the middle trunk. Each of the three trunks bifurcates into anterior and posterior divisions. The anterior divisions of the upper and middle trunks form the lateral cord. The anterior division of the lower trunk forms the medial cord. The posterior divisions of all three trunks form the posterior cord. ${ }^{[1,2]}$

The musculocutaneous nerve originates from the lateral cord of the brachial plexus. It usually supplies the coracobrachialis, biceps brachii and brachialis muscles in the anterior compartment of the arm and then continues as the lateral cutaneous nerve of the forearm. ${ }^{[3-5]}$ The median nerve is formed by two roots derived from the lateral and medial cords which embrace the second part of the axillar artery. If the lateral root is small, the musculocutaneous nerve connects with the median nerve in the $\operatorname{arm} .{ }^{[5]}$

The coracobrachialis is an elongated muscle in the superomedial part of the arm. It is a useful landmark for locating other structures in the arm; i.e., the musculocutaneous nerve pierces it, and the distal part of its attachment indicates the location of the nutrient foramen of the humerus. ${ }^{[0,7]}$

Variations in the formation of the brachial plexus and its terminal branches in the upper extremity are common and have been reported in the literature. ${ }^{[1]}$

Variations of the musculocutaneous nerve and its branches have also been reported previously. The musculocutaneous nerve is found to be absent or sometimes the nerve does not pierce the coracobrachialis muscle. ${ }^{[1,8-10]}$ It may adhere for some distance to the median nerve and pass behind the biceps. Some fibers of the median nerve may run in the musculocutaneous nerve, leaving it to join their proper trunk; less frequently the reverse occurs, and the median nerve sends a branch to the musculocutaneous nerve. ${ }^{[5,11]}$ When the musculocutaneous nerve is absent, branches from median nerve, after supplying all the muscles of front of arm, continue as the lateral cutaneous nerve of the forearm. ${ }^{[12]}$

Formation of the median nerve by two lateral and one medial root have been reported ${ }^{[13,14]}$ and communications between the musculocutaneous and median nerves are also well documented as the most frequent of all the variations. ${ }^{[5,8,15]}$ 
Innervation of the coracobrachialis muscle by a branch from the lateral root of the median nerve was reported. ${ }^{[3]}$ There also exists evidence of double heads of origin for the coracobrachialis muscle. ${ }^{[6,16]}$ These variations of the cords of brachial plexus and its terminal branches may present themselves clinically or may be observed during surgery. They are especially important for surgeons, anesthetists, neurologists, orthopedic surgeons and traumatologists during surgical exploration of the axilla and arm region to avoid damage to these nerves.

The objective of this study was to report the following variations which were found in a male cadaver dissected for the routine gross anatomy course: (1) the coracobrachialis muscle was pierced by two nerves, and (2) the unknown nerve after piercing coracobrachialis muscle joined the median nerve.

\section{Case Report}

During routine dissection of the neck, pectoral region, axilla and arm of the upper limb of a male cadaver of 48 years of age for medical students in the Department of Human Anatomy, GAMBY College of Medical Sciences, Bahir Dar, Ethiopia, we found a case which is rare in literature where the coracobrachialis muscle was pierced by two nerves: musculocutaneous and unnamed nerve, and the unnamed nerve joined the median nerve distal to the coracobrachialis muscle.

The dissection was performed according to the standard procedures of Cunningham's Manual of Practical Anatomy Vol. I that we use regularly to teach 1st year medical students in GAMBY College of Medical Sciences, Bahir Dar, Ethiopia. ${ }^{[17]}$ With the body in the supine position, all the necessary incisions were made on both upper extremities of the body to reflect the skin so as to expose the axilla, arm, and forearm. The axilla was carefully cleaned to examine the brachial plexus. Then, the skin, subcutaneous fat, and fascia were removed to expose the muscles, the nerves and the vessels of the axilla in anterior compartment of the arm and forearm.

Observation was done on the formation of the brachial plexus at the lateral, medial, posterior cords and branches of these cords below the clavicle. The branches of the cords in relation to the second part of the axillary artery were cleaned and observed.

After a careful observation, we found the following variation in the right upper extremity: the right median nerve was formed from two roots as usual, lateral and medial (the lateral root arised from the lateral cord and the medial root from the medial cord). Lateral to the lateral root of the median nerve, there existed a common sheath of nerves which split into two after a short distance, parallel to the formation of the median nerve - one as the musculocutaneous nerve laterally and the other unnamed nerve medially (Figures $\mathbf{1}$ and $\mathbf{2}$ ).

Both of the nerves pierced the coracobrachialis muscle in the middle part of the muscle, and the lateral one (musculocutaneous nerve) continued as the lateral cutaneous nerve of the forearm. On the other hand, the medial part (unnamed nerve) pierced the muscle in the middle, medial to the musculocutaneous nerve. This branch joined the median nerve distal to the muscle and it did not supply any other arm muscle (Figures 1 and 2).

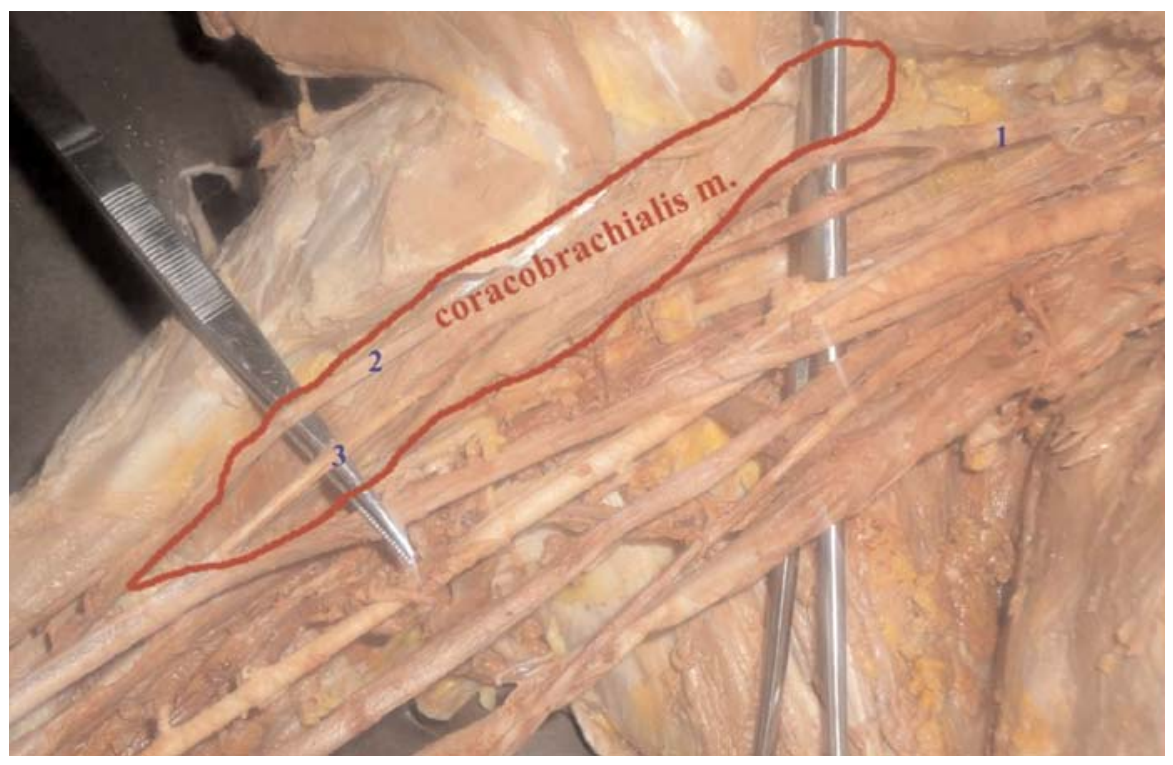

Figure 1. Right axilla and arm showing the coracobrachialis muscle pierced by two nerves. 1: common trunk for musculocutaneous and unnamed nerve; 2 : musculocutaneous nerve; 3 : unnamed nerve. [Color figure can be viewed in the online issue, which is available at www.anatomy.org.tr] 


\section{Discussion}

Variations in the formation, branching and distribution of the brachial plexus have been well-documented by many authors. Variations in the trunks, divisions and cord formation were observed. However, the makeup of the terminal branches were not affected. Variations in the nerve supply of the coracobrachialis muscle have also been explained by authors as mentioned earlier in this report. ${ }^{[1,5,8-12]}$ Appropriate knowledge of these variations other than those quoted in classical text books is important from both medical and surgical aspects.

Various authors reported communicating branches between the musculocutaneous and median nerves at different levels. Five types of variations ${ }^{[18]}$ were reported:

Type I: There is no communication between the median and musculocutaneous nerves.

Type II: Fibers of the medial root of the median nerve pass through the musculocutaneous nerve and join the median nerve in the middle of the arm.

Type III: The lateral root fibers of the medial root of the median nerve pass through the musculocutaneous nerve and, after some distance, leave it to form the root of the median nerve.

Type IV: The musculocutaneous nerve fibers join the lateral root of the median nerve and, after some distance, the musculocutaneous nerve arises from the median nerve.

Type V: The musculocutaneous nerve is absent and entire fibers of the musculocutaneous nerve pass through the lateral root, and fibers to the muscles supplied by a musculocutaneous nerve branch out directly from the median nerve.

Three different types of communication between the musculocutaneous and median nerves in relation to the coracobrachialis muscle ${ }^{[19]}$ were described:

Type I: The communication was proximal to the entrance of the musculocutaneous nerve into coracobrachialis muscle.

Type II: The communication was distal to the coracobrachalis muscle.

Type III: The nerve as well as the communicating branch did not pierce the coracobrachalis muscle.

Loukas and Aqueelah ${ }^{[20]}$ classified the communication patterns as follows:

Type I (45\%): The communications were proximal to the point of entry of the musculocutaneous nerve into the coracobrachialis muscle.

Type II (35\%): The communications were distal to the point of entry of the musculocutaneous nerve into the coracobrachialis muscle.

Type III (9\%): The musculocutaneous nerve did not pierce the coracobrachialis muscle.

Type IV (8\%): The communications were proximal to the point of entry of the musculocutaneous nerve and the coracobrachialis, and an additional communication took place distally.

Analysing our case in for these classifications, however, we found no communication between the musculocutaneous and median nerves; this coincides with Type 1 of

Figure 2. Right axilla and arm showing the coracobrachialis muscle pierced by two nerves, formation of the median nerve, and the union of an additional nerve to the median nerve distal to the muscle. 1: medial root of median nerve; 2: lateral root of median nerve; 3: median nerve; 4: common trunk for the musculocutaneous and unnamed nerves; 5: musculocutaneous nerve; 6: unnamed nerve; 7: ulnar nerve; 8: basilic vein; 9: medial cutaneous nerve of the arm; 10: posterior cord of the brachial plexus. [Color figure can be viewed in the online issue, which is available at www.anatomy.org.tr]

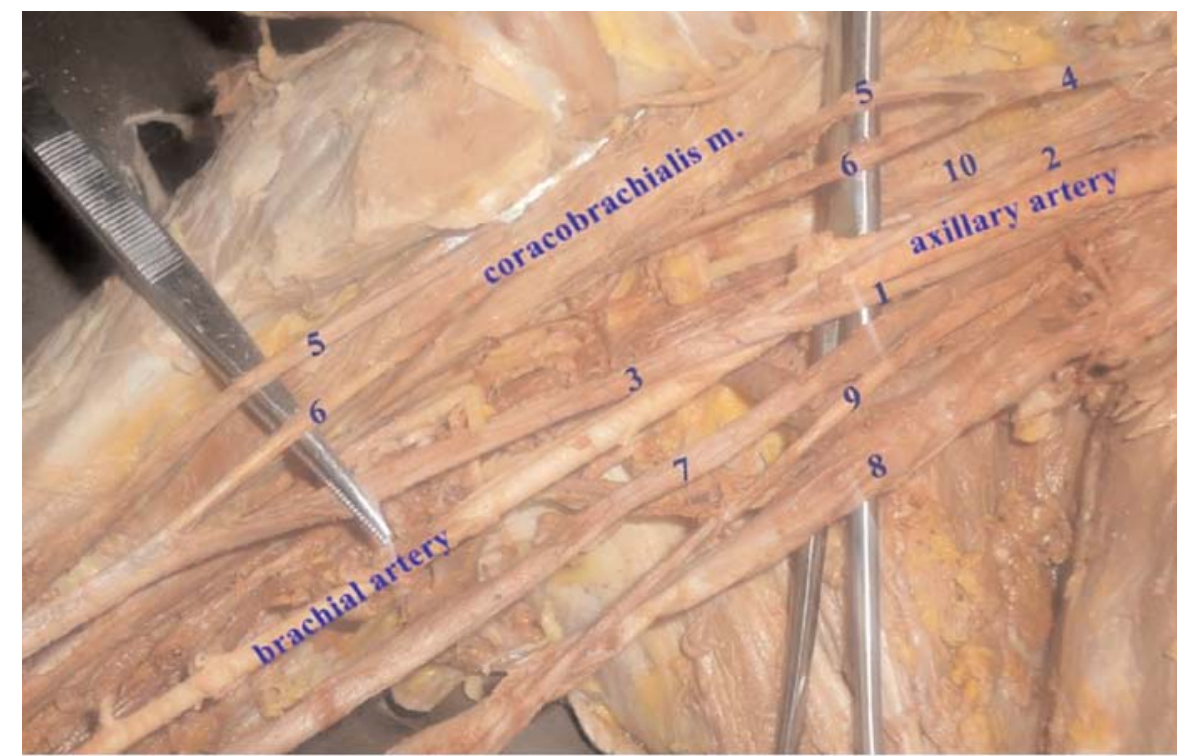


Le Minor's classification where there is no communication between the median and musculocutaneous nerves. ${ }^{[18]}$

Öztürk et al. ${ }^{[21]}$ reported a musculocutaneous nerve with three branches one of which ended in the coracobrachialis muscle. The second branch, piercing the coracobrachialis and giving its motor branches to the muscles of the anterior compartment of the arm, continued as the lateral cutaneous nerve of the forearm. The third branch also pierced the coracobrachialis muscle distal to the second branch and communicated with the median nerve. Similarly, in our case report there is a communication between the musculocutaneous nerve and the unnamed nerve proximal to the coracobrachialis muscle, lateral to the lateral root of the median nerve and also there exists a communication between the median nerve and the unnamed nerve distal to the coracobrachialis muscle.

Embryologically, variations in the formation, branching and distributions of the brachial plexus can be explained as follows: The limb muscles develop from the mesenchyme of the paraxial mesoderm during the fifth week of intrauterine life. The axons of the spinal nerves grow distally to reach the muscles and skin. Thus, a lack of coordination between these two processes due to altered signaling may lead to the development of multiple variations. ${ }^{[1,11]}$ The guidance of the developing axons is regulated by expression of chemoattractants and chemorepulsants in a highly coordinated site specific fashion. Alterations in the signaling between mesenchymal cells and neuronal growth cones or circulatory factors at the time of fusion of brachial plexus cords cause these variations." ${ }^{[2]}$ The existence of this anomaly may also be attributed to random factors influencing the mechanism of formation of limb muscles and peripheral nerves during embryonic life.

\section{Conclusion}

It is very important to be aware of the variations of the coracobrachialis muscle, formation of the brachial plexus, its terminal branches and its relation to surrounding structures during repair of brachial plexus lesions, shoulder arthroscopy and various other surgical and exploration procedures of the arm. Knowledge of these variations contributes to the existing information of the variations in the anatomy of the brachial plexus, explaining its morphological and clinical significance.

\section{Acknowledgements}

We would like to acknowledge GAMBY College of Medical Sciences, Bahir Dar, Ethiopia and first year medical students of the college.

\section{References}

1. Havaldar PP, Gupta A, Rajasekhar HV. Case report: variation in the formation of cords of brachial plexus and its relation with axillary artery. Current Neurobiology 2012;3:7-9.

2. Chaware PN, Ughade JM, Pandit SV, Maske GL. Variant branches of brachial plexus - a case report. International Journal of Anatomical Variations 2012;5:5-7.

3. Tatar I, Brohi R, Sen F, Tonak A, Celik H. Innervation of the coracobrachialis muscle by a branch from the lateral root of the median nerve. Folia Morphol (Warsz) 2004;63:503-6.

4. Bhattarai C, Poudel PP. Unusual variation in musculocutaneous nerves. Kathmandu Univ Med J 2009;7:408-10.

5. Gangadhara MS, Shakunthala Rao N, Krishna Kishore G, Manivannan K, Krishna Rao HR. Lower level of formation of lateral cord, variable formation of median nerve and communications between median and musculocutaneous nerve. International Journal of Biomedical and Advance Research 2014;5:464-7.

6. Moore KL, Dally AF, Agur AMR. Clinically oriented anatomy. 6th ed. Philadelphia (PA): Lippincott Williams and Wilkins; 2010. p. 738.

7. el-Naggar MM. A study on the morphology of the coracobrachialis muscle and its relationship with the musculocutaneous nerve. Folia Morphol (Warsz) 2001;60:217-24.

8. Jamuna M, Amudha G. A cadaveric study on the anatomic variations of the musculocutaneous nerve in the infraclavicular part of the brachial plexus. Journal of Clinical and Diagnostic Research 2011;5: 1144-7.

9. Ajayi NO, Lazarus L, Satyapal KS. Multiple variations of the branches of the brachial plexus with bilateral connections between ulnar and radial nerves. International Journal of Morphology 2012; 30:656-60.

10. Bhowmik K, Sarkar A. Clinically significant unilateral variation of musculocutaneous nerve. International Journal of Current Research and Review 2014;6:75-9.

11. Vollala VR, Potu BK, Gorantla VR, Reddy S. Variant median nerve and lateral antebrachial cutaneous nerve associated with anomalous brachial vein: case report. Neuroanatomy 2008;7:28-32.

12. Srimani P, Meyur R, De Bose A, Sadhu A. Unilateral variation of branches of brachial plexus supplying anterior compartment of arm. International Journal of Anatomical Variations 2014;7:112-4.

13. Satyanarayana N, Reddy CK, Sunitha P, Jayasri N, Nitin V, Praveen G, Guha R, Datta AK, Shaik MM. Formation of median nerve by three roots: a case report. Journal of College of Medical SciencesNepal 2010;6:47-50.

14. Talhar S, Sontakke BR, Bokariya P, Tarnekar AM, Shende MR. Bilateral variation in formation of median nerve. IOSR Journal of Pharmacy 2012;2:5-7.

15. Beheiry EE. Anatomical variations of the median nerve distribution and communication in the arm. Folia Morphol (Warsz) 2004;63: 313-8.

16. Gunenc Beser G, Ercakmak B, Tunali S, Basar R. Combination of six variations in a single arm. Rom J Morphol Embryol 2013;54: 845-9.

17. Romanes GJ. Cunningham's manual of practical anatomy. Volume 1: upper and lower limbs. Oxford: Oxford University Press; 2000. p. $46-53$. 
18. Le Minor JM. [A rare variation of the median and musculocutaneous nerves in man]. Arch Anat Histol Embryol 1990;73:33-42.

19. Venieratos D, Anagnostopoulou S. Classification of communications between the musculocutaneous and median nerves. Clin Anat 1998; $11: 327-31$.

20. Loukas M, Aqueelah H. Musculocutaneous and median nerve connections within, proximal and distal to the coracobrachialis muscle. Folia Morphol (Warsz) 2005;64:101-8.

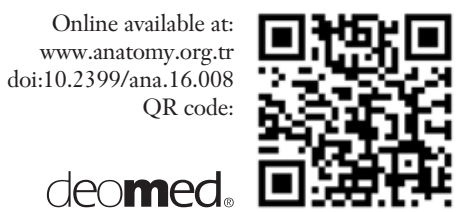

21. Öztürk NC, Uzmansel D, Öztürk H. An unreported pattern of musculocutaneous and median nerve communication with multiple variations of biceps brachii: a case report. Surg Radiol Anat 2010;32: 887-90.

22. Balachandra N, Kulkarni V, Ramesh BR. Absence of musculocutaneous nerve - a study of its incidence and clinical implications. International Journal of Basic and Applied Medical Sciences 2015; $5: 42-7$.

Correspondence to: Dawit Habte Woldeyes, MSc

Department of Human Anatomy, College of Medicine and Health Sciences, Bahir Dar University, P.O. Box 79, Bahir Dar, Ethiopia Phone: +251938221383

e-mail: dwthabte@gmail.com

Conflict of interest statement: No conflicts declared.

This is an open access article distributed under the terms of the Creative Commons Attribution-NonCommercial-NoDerivs 3.0 Unported (CC BY-NCND3.0) Licence (http://creativecommons.org/licenses/by-nc-nd/3.0/) which permits unrestricted noncommercial use, distribution, and reproduction in any medium, provided the original work is properly cited. Please cite this article as: Woldeyes DH, Abegaz BA. Coracobrachialis muscle pierced by two nerves: case report. Anatomy 2016;10(2):148-152. 